\title{
Sentimental Analysis for Social Media - A Review
}

\author{
Ms. R. Mahalakshmi \\ Assistant Professor, Department of Computer Engineering, NPA Polytechnic College, Kotagiri- 643217 \\ Email: mahasena2009@gmail.com \\ Dr. M. Nandhini \\ Assistant Professor, Department of Computer Science, Government Arts College, Udumalpet-642126 \\ Email:nandumano@gmail.com \\ Ms. G. Kowsalya \\ Research Scholar, Department of Computer Science, Government Arts College, Udumalpet-642126 \\ Email: kowsi.ganeshan@gmail.com
}

ABSTRACT-

In recent times, Social media has emerged as a personal communication media, as well as, a media to convey reviews about items and benefits or even political and general occasions among its clients. By using web and the web 2.0 the information in Twitter, Facebook and Instagram are easily retrieved. Because of its use across the board and prevalence, there is a monstrous measure of client surveys or feelings delivered and shared day by day. Mining client sentiments from Social Media is definitely not a straight forward assignment; it can be proficient in various ways. Gathering client sentiments can be costly and tedious assignment utilizing traditional strategies. This paper examines the challenges in doing sentimental analysis for Social Media.

Keywords - Data mining, Internet, Social Media, Sentimental analysis, Opinion mining.

Date of Submission: Aug 17, 2018

Date of Acceptance: Sep 07, 2018

\section{INTRODUCTION}

Internet based life have turned marvel due to the enormous and fast advances in data innovation. Individuals are utilizing online networking on consistent schedule to discuss their opinions with each other about wide assortment of subjects, items and administrations, which has made it a rich asset for text mining and notion investigation. Social Media(SM) communications incorporate Facebook, Twitter, and numerous others. Twitter is a standout amongst the most generally utilized online networking sites. In the Literature, there is no standard strategy for mining and investigating social media business information. A contextual investigation of two products can be exhibited to demonstrate the significance of examining client created online reviews from Microblogs. This is useful for business decision by observing reviews or opinions from client point of view.

\section{BACKGROUND}

In the recent decade, SM has turned out to be prominent and widespread communication media which shrinks the world in our packet. It helps in publicizing the products based on the individuals experience about precise items/administrations. This is said to be because of the efficient retrieval of information in the community sites through the use of web 2.0 innovations. Many persons are occupied with and depend on the SM for information, breaking reports and other varied topics. Clients find out what other person's view about any industry product, movie, education system, or significantly more authentic issues like look for other person's political view [1]. A large number of individual's are interested in SM data, for example, Twitter, Facebook, LinkedIn, YouTube and MySpace to scan out for data, breaking information and news refreshes. An outsized section of the updates are repeatedly posted by novel folks they have certainly not contact with other individuals. Therefore data assembled on SM is once in a while used to settle on profitable choices. While a few gatherings are recovering data from SM, others are redistributing information for the exploitation of other media clients [1]. The course of information transmit on the SM is extensive. So the systems are large. Likewise information sent by $\mathrm{SM}$ is enormously speedy and require data mining strategies to mine them for exactness and ease.

SM has given an imaginary strength on their customers by means of communicating their views and assumptions on SM sites, be it positive or negative [1]. Today, Organizations are aware of the importance of the viewpoint of consumers to the development of their goods or services. Personalities are being conscious of how they are seen by SM and attempts deeds to secure their image. Associations, political gatherings and even private people take after the exercises on the SM to stay up-to-date with how their group of spectator responds to issues that worries them.

\section{SENTIMENTAL ANALYSIS}

Sentiment analysis is the mechanized technique of understanding an opinion about a given subject from the created/commented posts or talked lingo. Sentimental analysis is done after clarifying the facts of SM. In [2], [3] sentimental analysis is carried out, by gathering opinions through SM clients to analyze market estimation. This 
helps the customers in choosing the products and services in market. Sentiment analysis can be alluded to as revelation and acknowledgment of negative or positive expression of opinion by individual persons on differing topics of intrigue. It is worthy to note that the tremendous opinions of a large number of SM clients are overpowering, running from vital ones to insignificant attestations [4]. Considerably, with the help of data mining systems, it has turned to be important in examining opinions to develop decision support system. This can be made possible by utilizing data mining algorithms and techniques to discover conclusion for the concerned topic, text, record or identity under survey. The idea behind sentiment analysis is basically to recognize potential drift in the society as it concerns the attitudes, observations, sentiment and the expectations of stakeholder or the public and to make necessary decisions. It is more critical to make an interpretation of opinion/sentiments communicated in SM and transformed into knowledge by using data mining techniques [5]. In this regard, data mining systems used in sentimental analysis of SM is reviewed in this paper.

In SM, the sentiment of the SM clients may be a decision, mood or assessment of a subjected object (product, movie, etc). It is classified as a positive or negative, in which, classification of sentiment, is a difficult task. Evaluation of sentiments can be done in two ways:

- Direct opinions: Directly giving positive or negative sentiments about the product.

- Comparison: Sentiments are submitted indirectly by comparing the subjected object with other similar objects.

Steps involved in sentiment analysis are given in fig 1.

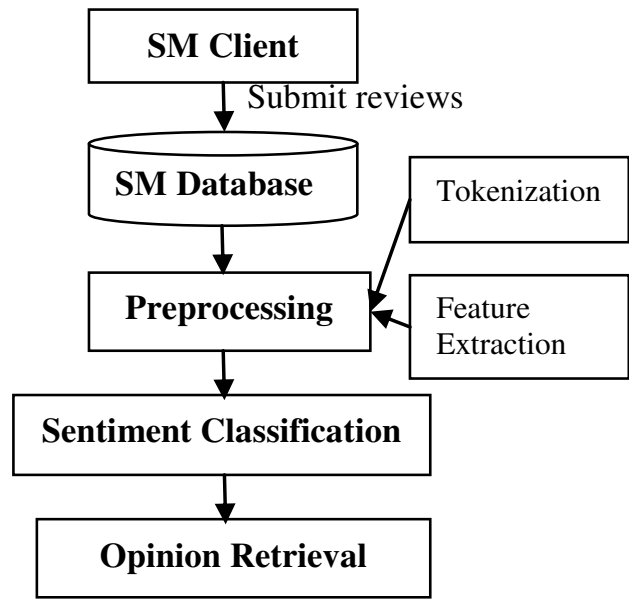

Figure 1. Sentiment Analysis - workflow

\section{THE STATE OF ART TECHNIQUES}

Sentiment analysis and Opinion Mining are essential for deciding opinions on brands and products, or understanding customers' state of mind. It is unrealistic to stay aware of the stream of new data by manual techniques. In the most recent decade, the field of automatically extracting knowledge has risen. There is a vast group of work carried on Opinion Mining for English. Comprehensively, two systems are utilized: Supervised learning and unsupervised learning. Supervised learning requires a training set of texts with physically appointed features and, from these cases, they learn the features (e.g. words) that correlate with the value. Unsupervised learning use a dictionary with words scored for extremity esteems, for example, impartial, positive or negative. Chaovalit and Zhou[6] assessed basic usage of the supervised and unsupervised systems and inferred that supervised procedures perform with around $85 \%$ exactness, though unsupervised strategies perform around $77 \%$. Supervised methods have the drawback that they require high quality training information for each kind of documents, for every domain and language. Unsupervised frameworks are stronger over various sorts of text and domains and, once the lexical and semantic resources are produced, can be deployed with less effort.

Other than the computational system that is utilized for Opinion Mining, there is an entire array of issues that play a role in the quality and usability of the opinion extraction. Most importantly, data mining can be connected to various levels of content: words, phrases, sentences, passages or reports. Words, as the littlest units, can have diverse polarities in various implications (e.g. "star" which can be objective as a glorious body or positive) as well as in various domains.

\section{RESEARCH ISSUES AND CHALLENGES}

Various research issues and difficulties in using data mining strategies in sentimental analysis are discussed below:

- The consequence of social strategies from information, and the issue of shielding individual privacy while investigating SM- While most research directed on SM utilize public information, rich source of social media information originates from emphatically ensured private information like email, telephone discussions and texting. Investigations are being directed on data chase, network creation [7],[8].

- The problem of link reference -Imagining the associations between different products are an issue in SM. By knowing those associations psychological militants are increased in SM.[9]

- Issues of valence while ordering sentiment - Even though sentiment analysis is about both positive and negative feelings. Neutral is being the center of positive and must not be ignored as the incorporation of objective preparation of examples in learning the dissimilarities in positive and negative feelings [10]. In any case, in various researchers up until this point, unbiased sentiment is unobserved while preparing classification models. [11]

- The problem of query classification [9].This issue arises because of information less questions and the indirect and biased goals of the clients. These issues provide a dialogue appetizer of how researchers will see straightaway the 
customer objective while utilizing the web to use information regarding customer inquiries. Grouping of inquiries is counted as a problem by scientists in the field [12], [13].

- Rating-inference - Going advance from categorizing texts or things based on positive or negative by using arithmetical mark (one star to five stars).

- Ambiguity in the opinion represented by customers Sentiment transmitted by analyst may once in a while be downy and twisty. It can be said as like neither there nor here. (e.g., "The Personal Computer is light-weight, the keys are elusive and easy to press, comes in lovely hues, still the charge is unreasonable; I can't normally declare if it's warranted, in spite of all the problem or not").

\section{MACHINE LEARNING TECHNIQUES}

Different frameworks are produced to identify/inspect the emotion rising from products, administrations, happening or character survey on SM [14]. There are plans publicly utilized as a part of assessment check, going from clearcut totaling methods to machine learning. This integrates categorizing opinion based on information utilizing balancing modification of optimistic against unconstructive.

The data mining tools discussed in this paper are being used which go starting form unsupervised, semisupervised to supervised learning.

- Supervised learning use structured data and/or potential human explanations as training set from which machines learn with a specific target to make future inferences.

- Semi and unsupervised machine learning do not use training set and machines must learn to interpret unstructured data with minimal or no guidance.

The result of frequent assessments in sentiment enquiry of SM has given handier to the instruction of SM [15]. It has likewise revealed the unique ways in analyzing the SM customers. Diverse data mining techniques are listed in Table 1

Table 1: Sentiment Analysis-Techniques

\begin{tabular}{|l|l|l|}
\hline \multicolumn{1}{|c|}{ Supervised } & Semi-supervised & \multicolumn{1}{c|}{ Unsupervised } \\
\hline $\begin{array}{l}\text { Support Vector } \\
\text { Machines(SVM) }\end{array}$ & $\begin{array}{l}\text { Transductive } \\
\text { SVM }\end{array}$ & $\begin{array}{l}\text { Sentiment } \\
\text { Orientation }\end{array}$ \\
\hline Naives Bayes & $\begin{array}{l}\text { Lexicon } \\
\text { classification }\end{array}$ & $\begin{array}{l}\text { Sentiment } \\
\text { Lexicon }\end{array}$ \\
\hline KNN & $\begin{array}{l}\text { Mincuts, } \\
\text { Randomized } \\
\text { Mincuts }\end{array}$ & k-means \\
\hline Neural Network & $\begin{array}{l}\text { Polarity } \\
\text { Detection }\end{array}$ & $\begin{array}{l}\text { Blank state } \\
\text { method based }\end{array}$ \\
\hline Decision Tree & $\begin{array}{l}\text { Graph Parts of } \\
\text { techniques }\end{array}$ \\
\hline
\end{tabular}

Sentiment analysis is rational mining of text which recognizes and removes biased data in resource objects, and helping a business to understand the social slant of their brand, item or organization. By using the ongoing advances in deep learning, the capacity of calculations to divide content has improved broadly. Creative utilization of cutting edge artificial intelligence procedures can be a forceful tool for achieving in detail research in sentiment analysis.

\section{CONCLUSION}

This paper examines the challenges of SM information which is mainly focused on opinions/sentiments communicated by SM clients. Data mining techniques made helpful consideration in the exploration did so far in this field. This is a clear capability of data mining has in managing enormous and self-motivated information. Distinctive authors have developed few computations that can be used to mine the sentiments of SM clients. Further, this paper gave an outline of the sentimental analysis and its challenges.

\section{REFERENCES}

[1] Pang, Bo, and Lillian Lee. "Opinion mining and sentiment analysis". Foundations and Trends ${ }^{\circledR}$ in Information Retrieval, 2(1-2), 2008, 1-135.

[2] Das, Sanjiv, and Mike Chen. "Yahoo! for Amazon: Extracting market sentiment from stock message boards." Proc. of the Asia Pacific Finance Association Annual Conf. (APFA). 35, 2001,43.

[3] Tong, Richard M. "An operational system for detecting and tracking opinions in on-line discussion." In Working Notes of the ACM SIGIR 2001 Workshop on Operational Text Classification, 1(6), 2001.

[4] Kaplan, A.M. and Haenlein, M., "Users of the world, unite! The challenges and opportunities of Social Media”. Business Horizons, 53(1), 2010, 59-68.

[5] Kaschesky, M., Sobkowicz, P. and Bouchard, G.,. "Opinion mining in social media: modeling, simulating, and visualizing political opinion formation in the web", Proc. $12^{\text {th }}$ Annual Int. Digital Government Research Conf.: Digital Government Innovation in Challenging Times, 2011, 317-326.

[6] Chaovalit, P. and Zhou, L. "Movie review mining: A comparison between supervised and unsupervised classification approaches", Proc. 38 ${ }^{\text {th }}$ Annual Hawaii International Conference on System Sciences (HICSS'05), 2005, 112c-112c.

[7] Kearns, Michael, Siddharth Suri, and Nick Montfort. "An experimental study of the coloring problem on human subject networks." Science, 313(5788), 2006, 824-827.

[8] Kleinberg, Jon. "Complex networks and decentralized search algorithms." Proc. Int. Congress of Mathematicians (ICM), 3, 2006, 1019-1044. 
Volume: 10 Issue: 03 Pages: 3860-3863 (2018) ISSN: 0975-0290

[9] Liben-Nowell, David, and Jon Kleinberg. "The link-prediction problem for social networks." Journal of the American society for information science and technology, 58(7),2007, 1019-1031.

[10] Koppel, M., and Schler, J. "The importance of neutral examples for learning sentiment". Computational Intelligence, 22(2), 2006,100-109.

[11] Qu, Yan. "Exploring attitude and affect in text: Theories and applications." AAAI Spring Symposium, 2004.

[12] Pang, Bo, and Lillian Lee. "Using very simple statistics for review search: An exploration." Coling 2008: Companion volume: Posters ,2008, 75-78.

[13] Pang, B. and Lee, L. "Seeing stars: Exploiting class relationships for sentiment categorization with respect to rating scales". Proc. $43^{\text {rd }}$ Annual meeting on association for computational linguistics, 2005,115-124.

[14] Godbole, Namrata, Manja Srinivasaiah, and Steven Skiena. "Large-Scale Sentiment Analysis for News and Blogs.", Icwsm, 7(21), 2007, 219-222.

[15] Cortizo J, Carrero F, Gomez J, Monsalve B, Puertas E. "Introduction to Mining SM". Proc. $1^{\text {st }}$ Int. Workshop on Mining SM, 2009, 1-3. 